This is an Open Access article, distributed under the terms of the Creative Commons Attribution licence (http://creativecommons.org/licenses/by/4.0/), which permits unrestricted re-use, distribution, and reproduction in any medium, provided the original work is properly cited.

doi:10.1017/jfm.2020.406

\title{
Phase separation effects on a partially miscible viscous fingering dynamics
}

\author{
Ryuta X. Suzuki ${ }^{1}$, Yuichiro Nagatsu ${ }^{1,4,}$, , Manoranjan Mishra ${ }^{2, \dagger}$ \\ and Takahiko Ban $^{3, \dagger}$ \\ ${ }^{1}$ Department of Chemical Engineering, Tokyo University of Agriculture and Technology, \\ 184-8588 Tokyo, Japan \\ ${ }^{2}$ Department of Mathematics, Indian Institute of Technology Ropar, 140001 Rupnagar, India \\ ${ }^{3}$ Division of Chemical Engineering, Department of Materials Engineering Science, Osaka University, \\ 560-8531, Osaka, Japan \\ ${ }^{4}$ PRESTO, Japan Science and Technology Agency, Saitama 332-0012, Japan
}

(Received 7 September 2019; revised 13 May 2020; accepted 19 May 2020)

Classical viscous fingering (VF) instability, the formation of finger-like interfacial patterns, occurs when a less viscous fluid displaces a more viscous one in porous media in immiscible and fully miscible systems. However, the dynamics in partially miscible fluid pairs, exhibiting a phase separation due to its finite solubility into each other, has not been largely understood so far. This study has succeeded in experimentally changing the solution system from immiscible to fully miscible or partially miscible by varying the compositions of the components in an aqueous two-phase system (ATPS) while leaving the viscosities relatively unchanged at room temperature and atmospheric pressure. Here, we have experimentally discovered a new topological transition of VF instability by performing a Hele-Shaw cell experiment using the partially miscible system. The finger formation in the investigated partially miscible system changes to the generation of spontaneously moving multiple droplets. Through additional experimental investigations, we determine that such anomalous VF dynamics is driven by thermodynamic instability such as phase separation due to spinodal decomposition and Korteweg convection induced by compositional gradient during such phase separation. We perform the numerical simulation by coupling hydrodynamics with such chemical thermodynamics and the spontaneously moving droplet dynamics is obtained, which is in good agreement with the experimental investigations of the ATPS. This numerical result strongly supports our claim that the origin of such anomalous VF dynamics is thermodynamic instability.

Key words: fingering instability, Hele-Shaw flows, multiphase flow

$†$ Email addresses for correspondence: nagatsu@cc.tuat.ac.jp, manoranjan@iitrpr.ac.in, ban@cheng.es.osaka-u.ac.jp 


\section{Introduction}

When a more viscous fluid is displaced by a less viscous fluid in a porous medium in immiscible or fully miscible fluid systems, it leads to the occurrence of Saffman-Taylor instability, resulting in the formation of finger-like interfacial patterns called viscous fingering (VF) (Homsy 1987). The nonlinear dynamics of classical $\mathrm{VF}$ is accepted to be governed by different mechanisms of shielding, spreading and splitting. Multiple fingers are initially formed owing to Saffman-Taylor instability, in which a finger slightly ahead of its neighbouring fingers shields them from further growth as a result of higher pressure gradient in the advancing finger, which grows further while spreading owing to interfacial tension. Tip splitting occurs when a finger reaches a certain width beyond a critical capillary number in an immiscible system, or a critical Péclet number in a miscible system (Homsy 1987). Various VF patterns can be formed, depending on the physicochemical properties of the fluids. Non-Newtonian properties induce branched fractal patterns (Nittmann, Daccord \& Stanley 1985) and fracturing patterns (Lemaire et al. 1991). Changes in interfacial tension, viscosity, viscoelasticity and permeability due to chemical reactions between the two fluids often create spiral, bending and filament-like fingering patterns (Fernandez \& Homsy 2003; Nagatsu et al. 2007, 2008a,b; Haudin et al. 2014) in experiments.

There is a quantitative difference between the fingering patterns in immiscible and fully miscible systems. The fingers in immiscible systems are significantly wider than those in fully miscible systems because interfacial tension stabilizes the fingering pattern (Chen 1989). Generally, fully miscible systems were considered not to comprise any interfacial tension (Paterson 1985). However, recent works have proved the existence of transient-effective interfacial tension in fully miscible systems. Several previous numerical studies have predicted the stabilizing effects of effective interfacial tension on fully miscible fingering dynamics (Chen, Wang \& Meiburg 2001; Pramanik \& Mishra 2013, 2015). Truzzolillo et al. (2014, 2016) experimentally investigated the effects of effective interfacial tension on the fingering patterns in two fully miscible fluids, a colloidal suspension and its own solvent. They rationally demonstrated that effective interfacial tension could stabilize the interfacial instability in fully miscible systems, as in immiscible systems. Very recently, several numerical studies have pointed out the importance of understanding the VF dynamics in partially miscible systems (Amooie, Soltanian \& Moortgat 2017; Fu, Cueto-Felgueroso \& Juanes 2017) mainly based on geological applications. However, the dynamics in partially miscible systems have not been largely understood so far. Especially, experimental approach on this issue is lacking.

In this study, we have succeeded in experimentally changing the solution system from immiscible to fully miscible or partially miscible by varying the concentrations of the components in an aqueous two-phase system (ATPS) while leaving the viscosities relatively unchanged at room temperature and atmospheric pressure. We have conducted VF experiments in a Hele-Shaw cell by using the ATPS. We have found anomalous VF dynamics giving rise to the spontaneously moving droplets, which has not been seen in classical immiscible or fully miscible systems. It is shown that such dynamics is observed due to the phase separation of spinodal type in the above ATPS (Ban, Aoyama \& Matasumoto 2010; Ban et al. 2012), which refers to the spontaneous phase separation of a mixture into coexisting phases induced by thermodynamic instability where the second derivative of the free energy with respect to composition is negative in binary mixture (Mauri, Shinnar \& Triantafyllou 1996). The partially miscible system in our study undergoes phase separation when the system is transferred into the spinodal region through direct contact between the more 


$\begin{array}{lccccc}\text { Solution } & \begin{array}{c}\mathrm{PEG} \\ \% \mathrm{w} / \mathrm{w}\end{array} & \begin{array}{c}\mathrm{Na}_{2} \mathrm{SO}_{4} \\ \% / \mathrm{w}\end{array} & \begin{array}{c}\text { Water } \\ \% \mathrm{w} / \mathrm{w}\end{array} & \begin{array}{c}\text { Viscosity } \\ \mathrm{mPa} \mathrm{s}\end{array} & \begin{array}{c}\text { Density } \\ \mathrm{g} \mathrm{cm}^{-3}\end{array} \\ 36.5 \% \mathrm{PEG} & 36.5 & 0 & 63.5 & 112 & 1.07 \\ 0 \% \mathrm{Na}_{2} \mathrm{SO}_{4} & 0 & 0 & 100 & 0.972 & 0.997 \\ 12 \% \mathrm{Na}_{2} \mathrm{SO}_{4} & 0 & 12 & 88 & 1.39 & 1.11 \\ 14 \% \mathrm{Na}_{2} \mathrm{SO}_{4} & 0 & 14 & 86 & 1.53 & 1.13 \\ 17 \% \mathrm{Na}_{2} \mathrm{SO}_{4} & 0 & 17 & 83 & 1.76 & 1.16 \\ 19 \% \mathrm{Na}_{2} \mathrm{SO}_{4} & 0 & 19 & 81 & 1.92 & 1.18 \\ 20 \% \mathrm{Na}_{2} \mathrm{SO}_{4} & 0 & 20 & 80 & 2.08 & 1.19 \\ \text { Phase L } & 36.5 & 3.2 & 60.4 & 125 & 1.08 \\ \text { Phase } \mathrm{H} & 1.4 & 16.0 & 82.5 & 1.76 & 1.16\end{array}$

TABLE 1. The viscosity and density of the solutions at $25^{\circ} \mathrm{C}$ with different $\%$ w/w used in the experiment.

viscous and the less viscous solutions instead of quenching since phase separation is limited to the mixing region. Thereby, throughout the paper the term 'phase separation' is used intermittently for the spinodal decomposition type of thermodynamic instability. Experimental results are compared with the theoretical findings with a viewpoint of coupling between hydrodynamics and chemical thermodynamics.

\section{Solution systems and experimental set-up}

The experiment is conducted using a radial Hele-Shaw cell, which consists of two parallel glass plates $\left(140 \times 140 \mathrm{~mm}^{2}\right)$ with a thin $(0.3 \mathrm{~mm})$ gap. An ATPS consisting of poly(ethylene) glycol (PEG) (weight-average molecular weight, $M w=8000$ ), sodium sulphate $\left(\mathrm{Na}_{2} \mathrm{SO}_{4}\right)$ salt and deionized water is used (see table 1) (Ban et al. 2012). Three types of experiments are performed with immiscible, fully miscible and partially miscible fluids. In the immiscible experiment, the less viscous liquid of phase $\mathrm{H}$ displaces the more viscous liquid of phase $\mathrm{L}$. The deionized water displacing the more viscous solution of $36.5 \%$ PEG corresponds to the fully miscible fluids. However, in other experiments, more viscous solution of $36.5 \%$ PEG displaced by the less viscous solution composed of different $\% \mathrm{w} / \mathrm{w}$ of salt $\left(C_{s}\right)$ are considered. At the interfacial region, the concentration of the solutions can become $18.25 \%$ PEG and half of the $\% \mathrm{w} / \mathrm{w}$ of salt and can fall into either region I (single phase) or region II (two phases) of the phase diagram of figure 1 (Snyder, Cole \& Sziag 1992). When the interface of the two solutions is in region II, it goes under the phase separation with salt-rich and PEG-rich phases, which is called partially miscible fluid zone. For instance, when PEG $=36.5 \%$ and $C_{s}=0 \%$ (deionized water, $\diamond$ in figure $1 a$ ), the concentration in the interfacial region becomes $18.25 \%$ PEG and $0 \%$ salt $(\star$ in figure $1 a$ ), which lies in one phase region and the system becomes fully miscible. In contrast, when $C_{s}=20 \%$, the concentration in the interfacial region becomes $18.25 \%$ PEG and $10 \%$ salt ( $\square$ in figure $1 a$ ), which is in region II and the system would take phase separation with salt and PEG-rich solutions due to spinodal decomposition. Therefore, the system is called partially miscible because the system is in the thermodynamically unstable zone. When a solution consisting of $13 \%$ $\mathrm{Na}_{2} \mathrm{SO}_{4}$ and $10 \%$ PEG is prepared ( $\triangle$ in figure 1), the solution eventually separates into two immiscible phases as PEG-rich $\mathrm{L}$ and salt-rich $\mathrm{H}$ (see figure 1a). Hence, it is observed that the ATPS can be moderated from immiscible to fully miscible 


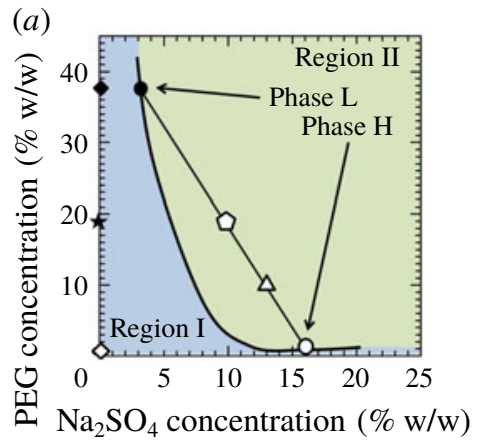

(b)

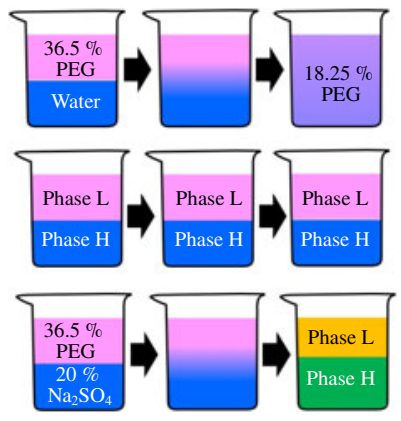

FIgure 1. (a) Phase diagram of PEG $8000-\mathrm{Na}_{2} \mathrm{SO}_{4}$ - water system (Snyder et al. 1992). (b) The schematics of the fully miscible, immiscible and partially miscible interfaces are indicated in the top, middle and below panels, respectively.

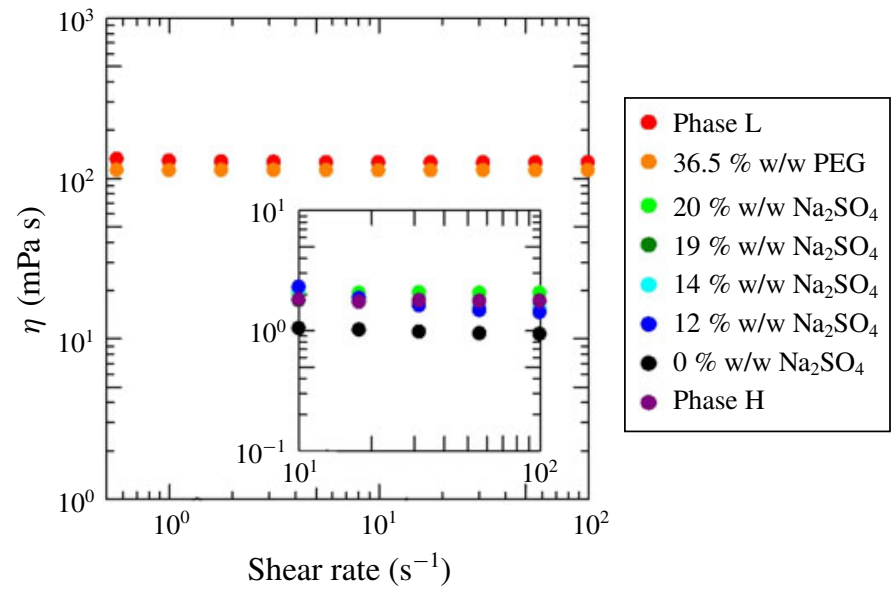

FIGURE 2. Shear viscosity measurement for different solutions.

or partially miscible by changing the concentrations using the ATPS at $25^{\circ} \mathrm{C}$ and atmospheric pressure by maintaining the viscosities of more and less viscous fluids quite similar.

Here, it should be emphasized that we confirm the solutions are Newtonian by performing bulk shear viscosity measurements (see figure 2) using a rheometer (AR-G2 TA instruments) of all the solutions considered in our analysis. It clearly shows that the viscosity remains constant with the variation of shear rate, and the first normal stress difference, one of viscoelastic properties, is not detected for all the solutions given in table 1 . These measurements signify the solutions are Newtonian although PEG has high molecular weight. In all the experiments, the more viscous liquid is dyed blue using indigo carmine to visualize the displacement patterns and we found that indigo carmine is insoluble in the $\mathrm{Na}_{2} \mathrm{SO}_{4}$ solution because of the salting-out effect. 


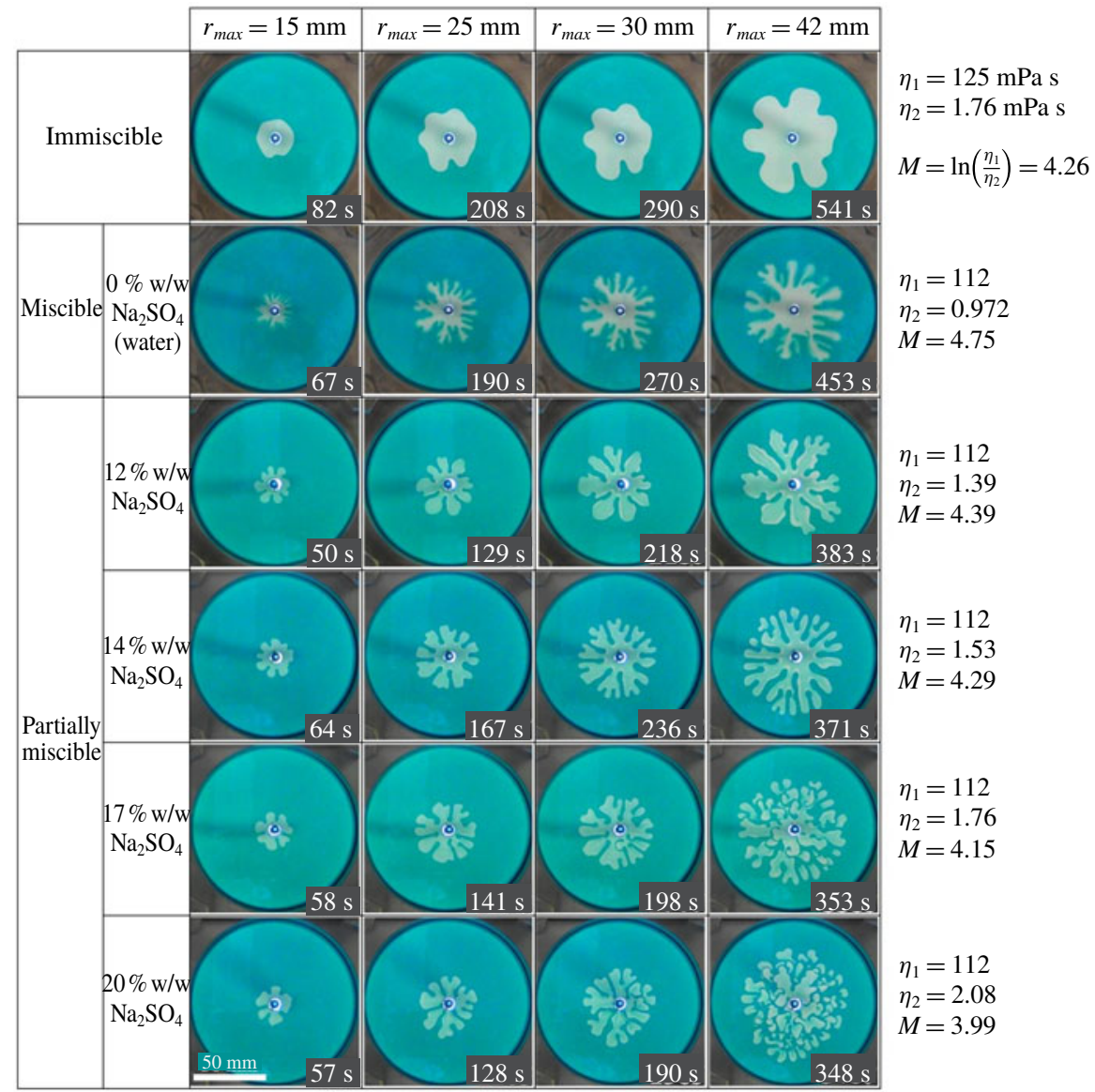

FIGURE 3. Displacement patterns with immiscible (phases $\mathrm{L}$ displaced by phase $\mathrm{H}$ in first row panel), fully miscible (second row panel with $0 \% \mathrm{w} / \mathrm{w} \mathrm{Na}_{2} \mathrm{SO}_{4}$ ) and other below panels are with $12,14,17,20 \%$ w/w of $\mathrm{Na}_{2} \mathrm{SO}_{4}$ for a fixed flow rate $q=$ $1.9 \times 10^{-9} \mathrm{~m}^{3} \mathrm{~s}^{-1}$. The patterns are shown at a different radius $r_{\max }$ in which the longest finger reached the radius and the injection time is shown in the lower right corner. The viscosity of the different solutions along with their log-mobility ratio is given in the right side of each panel, in which the viscosity $\eta_{1}$ corresponds to the more viscous solution which is displaced by the less viscous solution with viscosity $\eta_{2}$.

\section{Experimental results and discussions}

\subsection{Viscous fingering dynamics}

Figure 3 shows the evolution of viscous fingering dynamics for the immiscible, fully miscible and partially miscible systems (with $C_{s}=12,14,17$, and $20 \%$ ) for a fixed flow rate $q=1.9 \times 10^{-9} \mathrm{~m}^{3} \mathrm{~s}^{-1}$. Classical VF behaviour for the immiscible and fully miscible systems is observed and wider fingers are obtained in the immiscible than in the fully miscible case because of higher interfacial tension in the former. Surprisingly, in the partially miscible systems, we observe a multiple droplet formation pattern for $C_{s}=14,17$ and $20 \%$ (see supplementary movie file, available at https://doi.org/10.1017/jfm.2020.406). As shown in figure 3, before multiple droplets form, the interfacial distortion develops enough to allow 


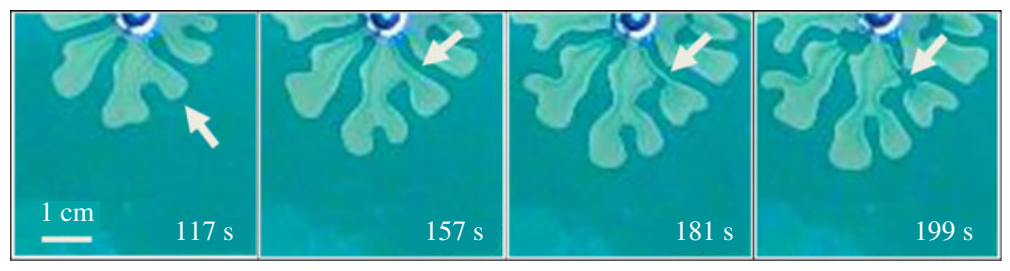

FIgURE 4. Temporal evolution of a finger with arrow marked to depict the pinched-off process, for $C_{s}=20 \%$ and $q=1.9 \times 10^{-9} \mathrm{~m}^{3} \mathrm{~s}^{-1}$.

the occurrence of the classical VF instabilities, such as spreading, shielding and splitting. Subsequently, the base of developed fingers pinches-off, which is clearly observed from figure 4 which depicts the process of a droplet formation through a time evolution of plots in a specific $20 \% \mathrm{w} / \mathrm{w}$ salt solution. For example, at $t=117 \mathrm{~s}$, classical viscous fingering phenomena like tip-splitting and shielding properties are seen and subsequently the shielded finger base becomes thinner (at $t=181 \mathrm{~s}$ ) and the droplet comes from the pinched-off finger at $t=199 \mathrm{~s}$ (see the pinched-off process with the arrow marked finger in figure 4). Eventually, multiple droplets detach from the mother viscous fingers. The detached droplets move anisotropically, and lead to a cascade of splitting (see figure 3 for corresponding 17 and $20 \%$ salt solution at $r_{\max }=42 \mathrm{~mm}$ ). From these observations, we claim that the secondary instability takes place as topological changes, leading to the formation of multiple droplets from the viscous fingers developed by Saffman-Taylor instability. Notably, although the steady value of interfacial tension between the PEG solution and the $17 \% \mathrm{Na}_{2} \mathrm{SO}_{4}$ solution is almost the same as that in the immiscible system, as described in $\S 3.2$, and both systems have the same viscosity ratio, the fingering patterns in the two systems are remarkably different. We observe that the length scale of droplet instability, such as droplet size, decreases with the increase in $C_{s}$ (see the last column panel of figure 3, with $C_{s}=14,17,20 \%$ ). In order to confirm this decreasing of the droplet size, we measured the average radius of the droplets as $R_{d, 42}=\sum_{n=1}^{N} \hat{R}_{d, 42, n} / N$ when $r_{\max }=42 \mathrm{~mm}$ for different salt solutions 17, 18, 19, 20\% (see figure 5), where $\hat{R}_{d, 42, n}=\sqrt{A_{d, 42, n} / \pi}$, index $n$ corresponds to a specific droplet number, $N$ is the total number of droplets, $A_{d, 42, n}$ is the area of the droplet $n$ corresponding to $r_{\max }=42 \mathrm{~mm}$. It is clearly found that the $R_{d, 42}$ decreases with the increasing of $C_{s}$. We measured this for the $C_{s}>17 \%$ as more droplets are formed in such ATPS. Interestingly, VF with no droplets is observed for $C_{s}=12 \%$, while for $C_{s}>13 \%$, multiple droplets begin to form. This occurs with the evidence that at the interfacial region for the $C_{s}=12 \%$ case, the solution is near the boundary curve of two different phases ( $18.25 \%$ PEG with $6 \%$ salt) of figure 1 . Hence, each salt solution with $C_{s}<13 \%$ will be in the one-phase system and $C_{s}>13 \%$ lies in the two-phase system giving rise to the droplet formation. It is to be noted that the viscosities of the solutions in the experiments are considered carefully in such manner that the log-mobility ratio, $M$, in all the cases remains close to each other.

\subsection{Hydrodynamic properties at fluid-fluid interface}

In order to understand the underlying hydrodynamic properties for the formation of droplet dynamics in the partially miscible system and to compare them with the immiscible and fully miscible interface patterns shown in figure 3 , we first 


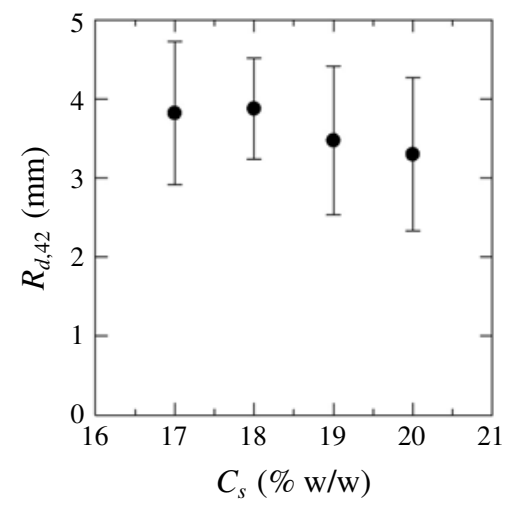

FIGURE 5. Average radius of the droplets $R_{d, 42}$ for different concentration of salt solutions $C_{s}$, which is measured corresponding to the $r_{\max }=42 \mathrm{~mm}$, i.e. last column of figure 3 .
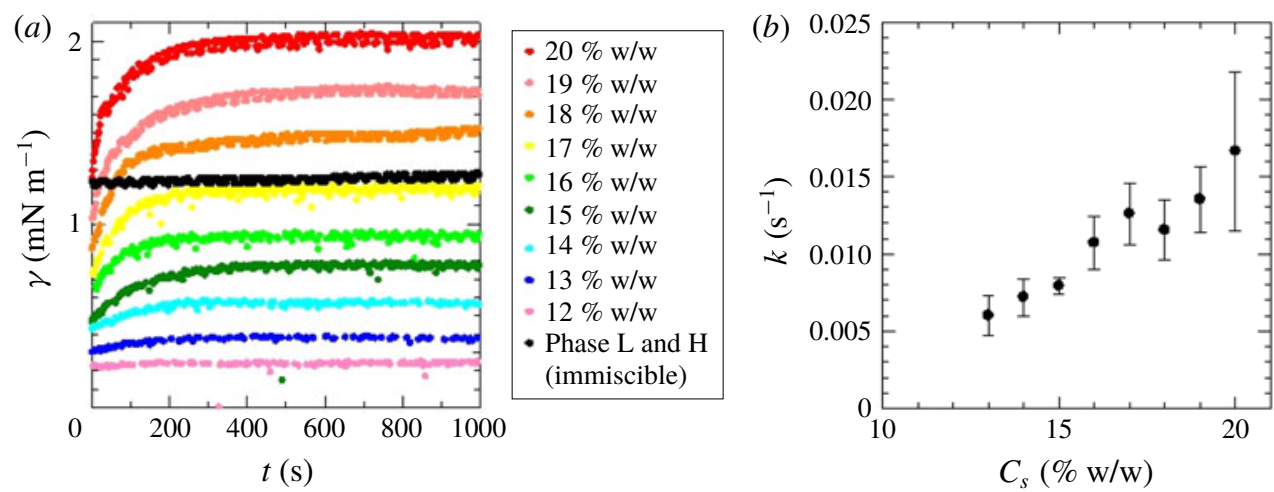

FIGURE 6. (a) Effective interfacial tension measurement versus time in a spinning drop tensiometer. (b) The phase separation rate constant $k$ versus salt concentration $C_{s}$. The error bars represent the average and standard deviation for three experiments.

measured the temporal change in effective interfacial tension, $\gamma$, between $36.5 \%$ PEG solution with different $C_{s}$ of $\mathrm{Na}_{2} \mathrm{SO}_{4}$ solutions. For measuring $\gamma$, a spinning drop tensiometer (SITE 100, Krüss, Germany) is used at $25^{\circ} \mathrm{C}$ with the rotation rate of the capillary of 6000 r.p.m. by following Pojman et al. (2006), and $\gamma$ is determined based on the shape, reflecting the balance between the interfacial and rotational energies. In the measurement, the PEG-rich solutions were dyed by indigo carmine in the same way as those in the VF experiment. In the immiscible system with the equilibrium compositions, as expected, $\gamma$ remained constant with time as shown in figure 6(a). The depicted temporal evolution of $\gamma$ between $36.5 \% \mathrm{w} / \mathrm{w}$ PEG solution and different salt solutions depending on $C_{s}$ shows that, $\gamma$ increased with time for $C_{s}>13 \%$ whereas it remained constant with time for $C_{s}=12 \%$. From a thermodynamic view, the increase in interfacial tension with time indicates the occurrence of a phase separation of spinodal type and the increase in interfacial energy to generate force (Ban et al. 2017). The force originating from such phase separation, which is called Korteweg force, tends to minimize the free energy stored in the interface and can generate convection (Vladimirova, Malagoli \& Mauri 1999; 
Ban et al. 2016). We claim that the concentration region where $\gamma$ increases with time corresponds to that where multiple droplet patterns were observed, shown in figure 3. Here, it is emphasized that such phase separation and Korteweg force play a crucial role in inducing such secondary instability of droplet formation. To consider an indicator of the rate of phase separation, we calculated the rate constant, $k$, from the time evolution of $\gamma$. The $k$ under the condition of $C_{s} \geqslant 13 \%$ was calculated by fitting the equation, $\gamma=\left(\gamma_{0}-\gamma_{\infty}\right) \mathrm{e}^{-k t}+\gamma_{\infty}$, where $\gamma_{0}$ and $\gamma_{\infty}$ are the values of interfacial tension at $t=0$ and at a steady state, respectively. The value of $k$ increases with $C_{s}$ (figure $6 b$ ). This result indicates that the rate of phase separation increases with $C_{s}$ and it is very much similar to the observation of smaller droplet size with increasing $C_{s}$ given in figure 5, which is important in understanding the anomalous VF dynamics in the partially miscible system, and further discussed in the next section.

\subsection{Measurement of area density and self-propulsion droplets dynamics}

Further, to uphold the existence of spinodal type phase separation with the salt concentration that falls in the two-phase zone of figure 1, we measured the temporal change in the area of the less-viscous salt-rich phase in both the immiscible and partially miscible systems during displacement. We consider only two salt concentrations, $C_{s}=17$ and $20 \%$ solution cases, and depict the evolution of area in figure $7(a)$, by using an image analysis software, ImageJ, to examine whether the phase separation occurred in the experiments or not. It is observed that the area of the injected salt-rich phase in the immiscible system is almost equal to the calculated area from the injection rate. However, the area in the partially miscible systems $\left(C_{s}=17\right.$ and $20 \%$ solutions) is larger than the areas of the injected salt-rich phase of the L-H system. The area difference clearly became pronounced over time (after $100 \mathrm{~s}$ ). We also verify that, when the mixture of PEG and salt solutions (with their respective concentrations) of equal volumes undergoes phase separation, the resulting volume of salt-rich phase becomes larger than the corresponding PEG-rich phase; this occurs as per the described phase diagram of figure 1. These results indicate the proof of the occurrence of phase separation in the partially miscible system during the displacement. In order to justify this, we have repeated the VF experiments for $C_{s}=17$ and $20 \%$ to find better statistics and calculated the temporal change of $\triangle A=A_{p m}-A_{i m}$, where $A_{p m}$ and $A_{i m}$ are the areas of the partially miscible $\left(C_{s}=17 \%\right.$ and $\left.20 \%\right)$ and immiscible systems, respectively. Further we have made the curve fitting using a least squares method for the averaged value of several experiments (three times for $C_{s}=17 \%$ and four times for $20 \%$ ) and observed that $\triangle A$ increases proportionally to $t^{1.4}$ for $17 \%$ (figure $7 b$ ) and $t^{1.7}$ for $20 \%$ (figure $7 c$ ). Such a power-law index is found with the coefficient of determination $R^{2} \sim 1$, i.e. for $C_{s}=17 \%, R^{2}=0.9968$ with $t^{1.4}$ and for $C_{s}=20 \%, R^{2}=0.9996$ with $t^{1.7}$. Such a coefficient of determination $R^{2}$ is a statistical measure to explain how well the regression predictions approximate the observed data points (Draper \& Smith 1998) and an $R^{2}$ of 1 indicates that the predictions perfectly fit the data. As known in the literature, if diffusion is the only driving force during phase separation, the typical size of a domain length scale increases with time as $t^{1 / 3}$ (Lifshitz \& Slyozov 1961), and if convection occurs owing to a balance of interfacial tension with viscous forces, the typical domain length grows linearly with time (Siggia 1979; Poesio, Beretta \& Thorsen 2009). But, in our case, we have obtained the area differences $\triangle A \propto t^{1.4}$ for $C_{s}=17 \%$ and $\triangle A \propto t^{1.7}$ for $C_{s}=20 \%$, which corresponds to the relation of length 

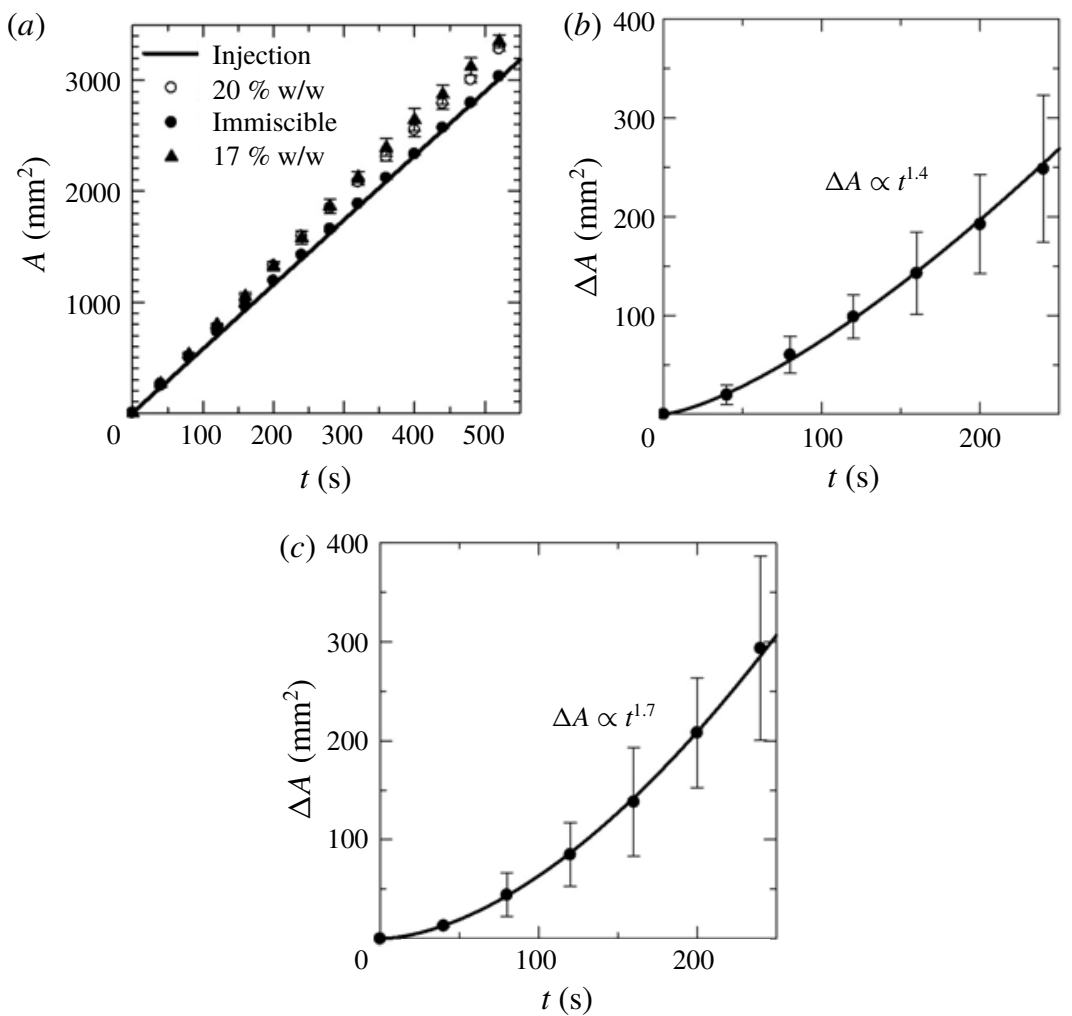

FIgURE 7. (a) Temporal change in the area of the salt-rich phase in both the immiscible and partially miscible systems during displacement at a flow rate $1.9 \times 10^{-9} \mathrm{~m}^{3} \mathrm{~s}^{-1}$. Temporal evolution of area difference $\triangle A$ between the salt concentrations and immiscible solutions with flow rate $1.9 \times 10^{-9} \mathrm{~m}^{3} \mathrm{~s}^{-1}$, and least square curve fitting shows $(b) \triangle A \propto$ $t^{1.4}$ with averaged value of several experiments for $C_{s}=17 \%$, and (c) $\triangle A \propto t^{1.7}$ for $C_{s}=20 \%$.

scale evolution with $t^{0.70}$ for $C_{s}=17 \%$ and $t^{0.86}$ for $C_{s}=20 \%$. The value of the index, 0.86 , is approximately close to 1 for $C_{s}=20 \%$, especially. This shows convection dominantly acts during phase separation as per the scaling argument given by Siggia (1979) and Poesio et al. (2009). However, the smaller value of the exponent as 0.70 for $C_{s}=17 \%$ is reasonable for the following reason: as the formation of the droplet is less for $C_{s}=17 \%$ than for $C_{s}=20 \%$, thus, the rate of phase separation and the effects of the spontaneous convention could be smaller for $C_{s}=17 \%$ than for $C_{s}=20 \%$.

In order to understand further the effects of Korteweg convection due to the phase separation, we performed an experiment of less viscous fluid with salt solution $C_{s}=19 \%$ displacing the more viscous fluid of $36.5 \%$ PEG solution in a flow rate $q=9.3 \times 10^{-10} \mathrm{~m}^{3} \mathrm{~s}^{-1}$. Just after several droplets formed, we stopped the injection. Interestingly, we observe that the droplets further move spontaneously. We measured the trajectories of several droplets after stopping the injection of the less viscous salt-rich liquid (shown in figure $8 a$ ) and calculated their mean squared displacements (shown in figure $8 b$ ). Since mean squared displacement (MSD) is powerful in distinguishing the dynamics of a moving object (Howse et al. 2007), we 
(a)

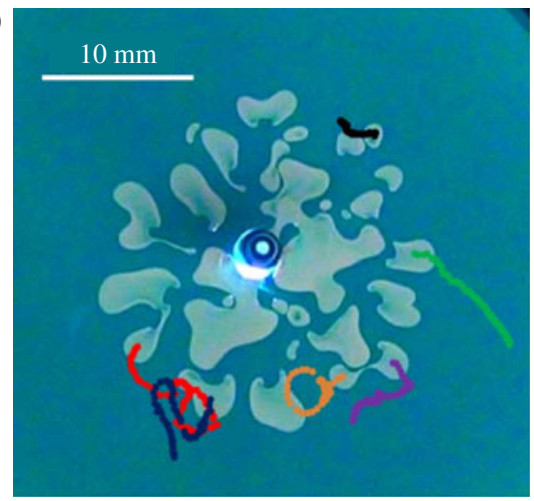

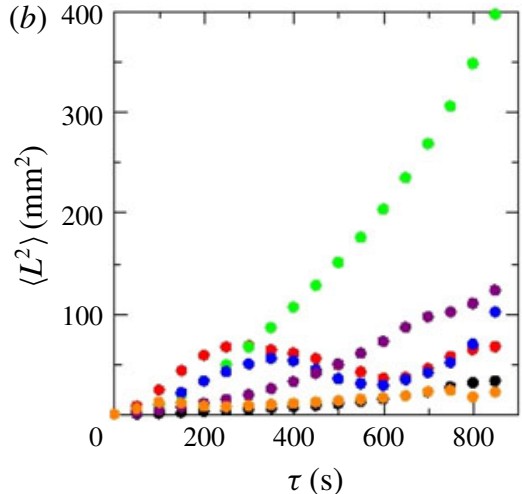

FIGURE 8. (a) The trajectories of six droplets after stopping the injection for $C_{s}=19 \%$ and $q=9.30 \times 10^{-10} \mathrm{~m}^{3} \mathrm{~s}^{-1} ;(b)$ mean squared displacements of the droplets calculated based on the trajectories shown in panel $(a)$. The colours of the dots correspond to those of the curves in the snapshots shown in panel $(a)$.

quantified the self-propelling dynamics through this MSD calculation. It is defined as

$$
\left\langle L^{2}\right\rangle=\frac{1}{N} \sum_{n=1}^{N}\left\{\left(x_{n}(t+\tau)-x_{n}(t)\right)^{2}+\left(y_{n}(t+\tau)-y_{n}(t)\right)^{2}\right\},
$$

where $x_{n}$ and $y_{n}$ are $x$ - and $y$-coordinates of the position of droplet, $t$ is elapsed time after stopping the injection, $\tau$ is the interval time and $N$ is the number of samples. Figure $8(b)$ clearly indicates that such droplets move at a constant speed initially and some droplets have reciprocatory motion; this is due to the oscillatory behaviour of the MSD. These results show that the formed multiple droplets can spontaneously propel even in the absence of fluid injection.

\subsection{Mechanism of the secondary instability}

The development of the second instability dynamics can be divided into two stages: formation of the droplets from the developed viscous fingers and self-propulsion of the droplets. The mechanism of droplet formation should be similar to the mechanism proposed by De Wit \& Homsy (1999). Their numerical study simulated fully miscible VF with a chemical reaction, the kinetics of which were essentially similar to a kind of phase separation, producing two fluids of low and high concentrations. When concentrations fell in the unstable region, the system spontaneously evolved toward the attracting chemical equilibrium. The convection generated by the variation of viscosity with the concentration enhanced system evolution. Both effects resulted in the pinching of viscous fingers and finally led to the formation of droplets. Our experimental system undergoes thermodynamic instability and phase separation of a spinodal type but without chemical reactions. In contrast to normal diffusion, such phase separation promotes mass transfer against the concentration gradients and enhances concentration fluctuations, leading to the separation of the system to low and high concentration regions. Furthermore, the body force generated by the compositional gradient creates a spontaneous fluid convection, promoting phase separation (Hohenberg \& Halperin 1977; Vladimirova et al. 1999). Thus, the 


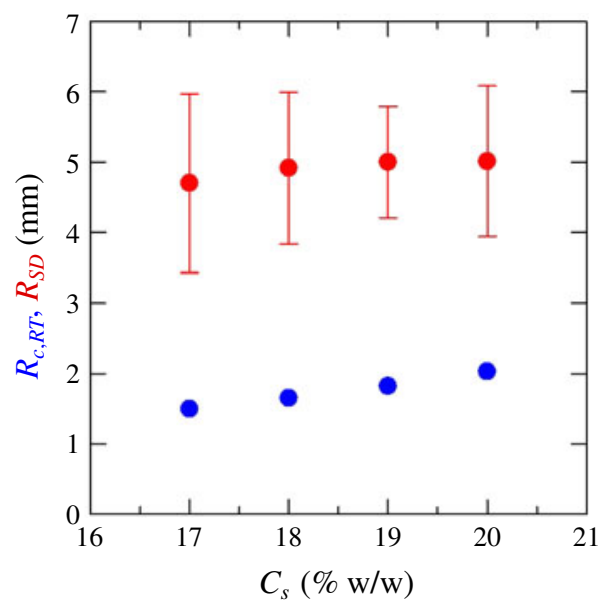

FIGURE 9. Average radius of the first generation droplets $R_{S D}$ due to the phase separation by spinodal decomposition comparing with the critical radius of the droplet due to Rayleigh-Taylor instability $R_{c, R T}$ for different concentrations of salt solutions $C_{s}$.

growth of the deformed region due to hydrodynamic instabilities is enhanced by the thermodynamic instabilities when the salt solution is in contact with the PEG solution in the spinodal region. Eventually, droplets are detached from the deformed region.

Furthermore, we find whether the droplet formation is attributed to buoyancy effects or Rayleigh-Taylor (RT) instability or not, by comparing the average first droplets formed in our experiment with the calculated critical radius, $R_{c, R T}$ due to RT instability, given as (Ban, Yamagami \& Furumichi 2012)

$$
R_{c, R T}=3.84 \sqrt{\frac{\gamma}{\triangle \rho g}},
$$

where $\gamma$ is an initial interfacial tension, $\Delta \rho$ is density difference between displacing and displaced solutions and $g$ is the gravitational acceleration $\left(9.807 \mathrm{~m} \mathrm{~s}^{-2}\right)$. The experimentally obtained radius of the droplet $R_{S D}$, which is due to the phase separation, and critical droplet size due to RT instability, $R_{c, R T}$, are plotted in figure 9. The $R_{S D}$ is the average radius of the first generation of the droplets formed in the ATPS of $17,18,19,20 \%$ and defined as $R_{S D}=\sum_{n=1}^{N} \hat{R}_{d, n} / N$, where $\hat{R}_{d, n}=\sqrt{A_{d, n} / \pi}, N$ is the total number of such first generation of droplets, $A_{d, n}$ is the area of the droplet $n$. It is observed that the average first detached droplets radius $R_{S D}$ is approximately $5.01 \mathrm{~mm}$ for $C_{s}=20 \%$, which is much larger than the critical radius, $R_{c, R T}$ predicted by RT instability, i.e. $1.99 \mathrm{~mm}$ for $C_{s}=20 \%$. Similarly, larger droplet radius is also observed as compared to the corresponding $R_{c, R T}$ for all other $C_{s}=17,18,19 \%$ solutions where eventually more droplets are formed. Hence, we confirm that the droplet formation in the partially miscible system is not from the buoyancy effects or the Rayleigh-Taylor instability. Regarding the self-propulsion of the droplets, this is the same as spontaneous motions of a single droplet by the Korteweg effect, which has been reported by several researchers, e.g. Ban et al. (2012, 2016), Molin, Mauri, \& Tricoli (2007) and Poesio et al. (2009).

To understand further that the considered ATPS (water-PEG-salt) undergoes phase separation, i.e. thermodynamically unstable with a specific composition of salt, the 
quantitative analysis is performed using the underlying Gibbs free energy per mole $\triangle G_{m}$ based on the well known Flory-Huggins equation (Sargantanis \& Karim 1997),

$$
\frac{\triangle G_{m}}{R T}=\phi_{w} \ln \phi_{w}+\frac{\phi_{p}}{r_{p}} \ln \phi_{p}+\frac{\phi_{s}}{r_{s}} \ln \phi_{s}+\chi_{p w} \phi_{p} \phi_{w}+\chi_{p s} \phi_{p} \phi_{s}+\chi_{s w} \phi_{s} \phi_{w},
$$

where $\phi_{i}$, with $i$ as $p, w$ and $s$ (PEG, water and salt, respectively) is the volume fraction and computed segment number $r_{p}=408.1, r_{s}=2.9$. The gas constant is $R$ and $T$ is the absolute temperature. The corresponding interaction parameters $\chi_{i j}$ between the $p, w$, and $s$ are $\chi_{p s}, \chi_{s w}, \chi_{p w}$ and measurement of such compositional dependent interaction parameters of the ATPS were only obtained experimentally, which was a very challenging task. Recently, Morita (2013) measured $\chi_{p s}, \chi_{s w}, \chi_{p w}$ successfully based on the cloud-point measurement using a refractometer (ATAGO, RX-7000i) following the procedure explained by Mohsen-Nia, Rasa \& Modarress (2006). Such a measurement is a technique of finding the temperature at which the sample of the mixture gets cloudy and then justifying the phase separation of the mixture compositions. From this cloud-point measurement the linear correlations, between $\chi_{i j}$ and $W_{p} / W_{w}$ (weight fraction of PEG with water), are obtained as $\chi_{p w}=0.670-1.01 W_{p} / W_{w}, \chi_{p s}=5.59-16.9 W_{p} / W_{w}, \chi_{s w}=8.22-15.9 W_{p} / W_{w}$. Using such $\chi_{i j}$ and calculating the corresponding $\phi_{p}, \phi_{w}, \phi_{s}$, we found that the $\partial^{2} \triangle G_{m} / R T / \partial C_{s}^{2}=$ $-6.17 \times 10^{-5},-7.47 \times 10^{-5},-9.14 \times 10^{-5},-10.2 \times 10^{-5},-10.7 \times 10^{-5} \mathrm{~J} \mathrm{~mol}^{-3}$ for $C_{s}=12,14,17,19,20 \%$, respectively. This result justifies that the interface undergoes thermodynamically unstable phase separation of spinodal type as the concentration conditions satisfy $\partial^{2} \triangle G_{m} / R T / \partial C_{s}^{2}<0$ (Porter, Easterling \& Sherif 2009; Kwiatkowski Da Silva et al. 2018) which shows they are in the spinodal region. Further spontaneous Korteweg convection drives the secondary instability dynamics of multiple droplet formation shown in figure 3 . However, in the case of $C_{s}=12 \%$ the effectiveness of phase separation is small as it falls near the boundary region of phase diagram shown in figure 1. Based on this, we claim that in the current study, the phase transformation is initially driven by diffusion, which is followed by such a phase separation which takes place locally in the interfacial region. We propose that this is a new interfacial dynamics with spinodal type phase separation. Such phase separation is also called one of uphill diffusion phenomena; see Krishna (2015) for a detail review on this subject and that arises due to the strong coupling between the diffusive fluxes of species and the thermodynamic forces due to chemical potential gradients.

Further, to compare with the experimental findings of the second part of the secondary instability, i.e. self-propelling droplets shown in figure $8(b)$, we perform the numerical simulations using the model equations of conservation of mass and momentum by neglecting the convective force of Stokes equations (Ban et al. 2016),

$$
\begin{gathered}
\boldsymbol{\nabla} \cdot \boldsymbol{v}=0, \\
\frac{\partial \phi_{i}}{\partial t}+\boldsymbol{v} \cdot \nabla \phi_{i}=-\frac{1}{\rho} \nabla \cdot \boldsymbol{j}_{i}, \\
\eta \nabla^{2} \boldsymbol{v}-\nabla p=-\sum_{i=p, s, w} \boldsymbol{F}_{i}, \\
\boldsymbol{F}_{\boldsymbol{i}}=\frac{\rho R T}{M_{w}} \mu_{i} \nabla \phi_{i},
\end{gathered}
$$

where $\boldsymbol{v}, \eta, p$ are the velocity, viscosity and pressure, respectively, and $\boldsymbol{j}_{\boldsymbol{i}}$ the diffusion flux of component $i$. Here, $\rho$ is the density, $M_{w}$ is the molecular weight, $\phi_{i}$ is the 

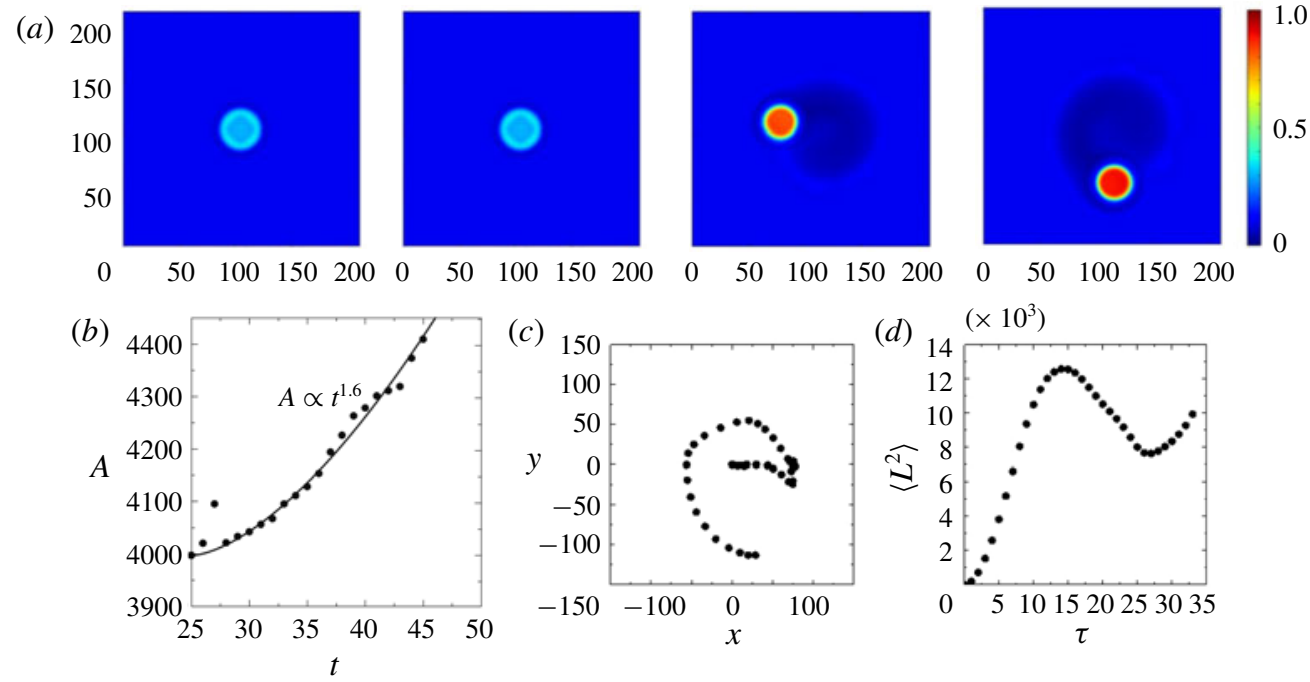

FIgURE 10. (a) Concentration field of $\phi_{p}$ obtained through numerical simulation. The dimensionless times are $t=1,20,30$ and 40 (from left to right). (b) Time evolution of droplet area $A$. (c) Trajectory of the computed droplet with initial composition in the drop $\left(\phi_{w}=0.5, \phi_{p}=0.3, \phi_{s}=0.2\right)$. It is formed in a uniform continuous phase with initial composition $\phi_{w}=0.1, \phi_{p}=0.1, \phi_{s}=0.8$ obtained from numerical simulation. $(d)$ Mean squared displacement of self-propelled droplet corresponding to panel $(c)$.

molar fraction of component $i$, and $\mu_{i}\left(=\delta G / \delta \phi_{i}\right)$ is the generalized chemical potential of component $i$. The body force $\boldsymbol{F}_{\boldsymbol{i}}$ is directed toward the rich region of component $i$, which is referred to as Korteweg force in the literature (Vladimirova et al. 1999; Ban et al. 2012). We numerically simulate the motion of the single self-propelled droplet through Korteweg force and the detailed procedure of numerical simulation is described in Ban et al. (2016). Figure 10(a) depicts, the concentration field of $\phi_{p}$ in the single droplet. Considering the respective free energy calculation of (3.1) with the above compositions and assuming the corresponding $\chi_{p w}=3, \chi_{s w}=3, \chi_{p s}=6$ for this numerical computation, we find the second derivative of Gibbs free energy with respect to the salt is also negative, i.e. in the thermodynamically unstable state. It is observed that, the droplet initially absorbs material from the surrounding continuous phase and excavates a ditch of material around its perimeter. The concentration profile around the droplet becomes non-uniform, thereby exerting body force $\boldsymbol{F}_{\boldsymbol{i}}$ leading to the spontaneous motion of the droplet at subsequent time. Also the area, trajectory and the MSD of the simulated moving droplet are shown in figures $10(b), 10(c)$ and $10(d)$, respectively. In the simulation, the area is defined as the region occupied by the molar fraction of the water $\phi_{w}>0.5$ and the initial concentration of water in the drop is $\phi_{w}=0.5$. At the early stage, $\phi_{w}$ decreases by diffusion since the initial molar fraction of the water in the surrounding is $\phi_{w}=0.1$. Thus, the area where the $\phi_{w} \geqslant 0.5$ decreases at the early stage. Therefore we show the area of the drop evolution after $t \geqslant 25$, where the area represents the existence of phase separation, in the figure $10(b)$. Interestingly, increasing of drop area is proportional to $t^{1.6}$ (figure 10b) and such dynamics qualitatively matches our experimental findings presented in figure $7(b)$, i.e. the area increases with a power index $\sim 1.7$ corresponding to the length increasing close to linear in time (Poesio et al. 2009), justifying the 
spontaneous convection during phase separation. Also, the numerical simulations clearly show the rotational motion of the droplets (see figure 10c) expressed by the oscillatory behaviour of the MSD in figure $10(d)$ signifying the self-propelling dynamics of the secondary instability. We emphasize that figure $10(d)$ matches our experimental results in figure $8(b)$. Since the simulation considers the body force term in the momentum equation, the agreement between the experiment and simulation demonstrates that the body force should play a role in the experiment.

Here, we also mention that, in our experiment, miscibility of two aqueous solutions is thermodynamically controlled by varying the salt concentrations, thereby allowing systematic changes in the interfacial tension and underlying dynamics. Since the concentration of PEG, which is a surface-active agent, almost remains constant in all of our experiments, the droplet formation is not driven by the Marangoni effect, rather it is due to spontaneous convection induced by the phase separation.

\section{Conclusion}

We have succeeded in experimentally changing the solution system from immiscible to fully miscible or partially miscible by varying the concentrations of the components in an aqueous two-phase system while leaving the viscosities relatively unchanged at room temperature and atmospheric pressure. We have experimentally discovered a new topological change of VF instability obtaining a secondary instability such as multiple moving-droplets formation in a partially miscible system. We have proved, using several experimental methods such as measurement of VF areas in Hele-Shaw flow, interfacial tension measurement through spinning drop tensiometer, and cloud point measurement, that such kind of secondary instability occurs due to the spinodal type phase separation in the ATPS mixture which generates a Korteweg convection by compositional gradient. Further, we have shown the comparison of experimental observation of self-propelling droplet motion with numerical simulation of the transport equation using a chemical thermodynamic model. To our knowledge, this is the first successful experiment to find such secondary instability in a partially miscible system and to depict how hydrodynamics and chemical thermodynamics (phase separation) interplay leads to anomalous topological pattern dynamics. Such a discovery contributes in opening a new area of research by coupling hydrodynamics and chemical thermodynamics, and can be very useful for future developments related to global warming and energy problems, in particular, $\mathrm{CO}_{2}$-EOR (Orr \& Taber 1984), because such techniques are based on fluid displacement processes in partially miscible systems. Also, various other possible biological applications like the formation of spontaneous lipid droplets (Thiam, Farese \& Walther 2013) and microfluidic single cell screening assays for the inhibition of a drug target (Debs et al. 2012; Schneider, Kreutz \& Chiu 2013) can be understood using the present coupling of hydrodynamics and chemical thermodynamics.

\section{Acknowledgements}

This work was supported by PRESTO-JST (no. 25103004 'Phase Interfaces for Highly Efficient Energy Utilization'). M.M. gratefully acknowledge the JSPS Invitation Fellowships for Research in Japan (no. L19548).

\section{Declaration of interests}

The authors report no conflict of interest. 


\section{Supplementary movie}

Supplementary movie is available at https://doi.org/10.1017/jfm.2020.406.

\section{REFERENCES}

Amooie, M. A., Soltanian, M. R. \& Moortgat, J. 2017 Hydrothermodynamic mixing of fluids across phases in porous media. Geophys. Res. Lett. 44, 3624-3634.

Ban, T., Aoyama, A. \& Matasumoto, T. 2010 Self-generated motion of droplets induced by Korteweg force. Chem. Lett. 39, 1294-1296.

Ban, T., Fukuyama, T., Makino, S., Nawa, E. \& Nagatsu, Y. 2016 Self-propelled vesicles induced by the mixing of two polymeric aqueous solutions through a vesicle membrane far from equilibrium. Langmuir 32, 2574-2581.

Ban, T., Kobayashi, Y., Suzuki, R. \& Nagatsu, Y. 2017 Active liquid matter driven by nonequilibrium interfacial tension. J. Phys. Soc. Japan 86, 101005.

Ban, T., Yamada, T., Aoyama, A., Takagi, Y. \& OKano, Y. 2012 Composition-dependent shape changes of self-propelled droplets in a phase-separating system. Soft Matt. 8, 3908-3916.

Ban, T., Yamagami, T. \& FurUmichi, Y. 2012 Transient pore dynamics in pH-responsive liquid membrane. Langmuir 28, 10682-10687.

Chen, C. Y., WAng, L. \& Meiburg, E. 2001 Miscible droplets in a porous medium and the effects of Korteweg stresses. Phys. Fluids 13, 2447-2456.

Chen, J. D. 1989 Growth of radial viscous fingers in a Hele-Shaw cell. J. Fluid Mech. 201, 223-242.

Debs, B. E., Utharala, R., Balyasnikova, I. V., Griffiths, A. D. \& Merten, C. A. 2012 Functional single-cell hybridoma screening using droplet-based microfluidics. Proc. Natl Acad. Sci. USA 109, 11570-11575.

De Wit, A. \& Homsy, G. M. 1999 Viscous fingering in reaction-diffusion systems. J. Chem. Phys. 110, 8663-8675.

Draper, N. R. \& Smith, H. 1998 Applied Regression Analysis, 3rd edn. Wiley.

FERnANdeZ, J. \& Homsy, G. M. 2003 Viscous fingering with chemical reaction: effect of in situ production of surfactants. J. Fluid Mech. 480, 267-281.

Fu, X., Cueto-Felgueroso, L. \& Juanes, R. 2017 Viscous fingering with partially miscible fluids. Phys. Rev. Fluids 2, 104001.

Haudin, F., Cartwright, J. H. E., Brau, F. \& De Wit, A. 2014 Spiral precipitation patterns in confined chemical gardens. Proc. Natl Acad. Sci. USA 111, 17363-17367.

Hohenberg, P. \& Halperin, B. 1977 Theory of dynamic critical phenomena. Rev. Mod. Phys. 49, 435-479.

Homsy, G. M. 1987 Viscous fingering in porous media. Annu. Rev. Fluid Mech. 19, 271-311.

Howse, J. R., Jones, R. A. L., Ryan, A. J., Gough, T., Vafabakhsh, R. \& Golestanian, R. 2007 Self-motile colloidal particles: from directed propulsion to random walk. Phys. Rev. Lett. 99, 048102.

KrishnA, R. 2015 Uphill diffusion in multicomponent mixtures. Chem. Soc. Rev. 44, 2812-2836.

Kwiatkowski Da Silva, A., Ponge, D., Peng, Z., Inden, G., Lu, Y., Breen, A., Gault, B.\& RAABE, D. 2018 Phase nucleation through confined spinodal fluctuations at crystal defects evidenced in Fe-Mn alloys. Nat. Commun. 9 (1), 1-11.

Lemaire, E., Levitz, P., Daccord, G. \& Van Damme, H. 1991 From viscous fingering to viscoelastic fracturing in colloidal fluids. Phys. Rev. Lett. 67, 2009-2012.

Lifshitz, I. M. \& Slyozov, V. V. 1961 The kinetics of precipitation from supersaturated solid solutions. J. Phys. Chem. Solids 19, 35-50.

Mauri, R., Shinnar, R. \& Triantafyllou, G. 1996 Spinodal decomposition in binary mixtures. Phys. Rev. E 53, 2613-2623.

Mohsen-Nia, M., RASA, H. \& ModarRess, H. 2006 Cloud-point measurements for (water + poly(ethylene glycol) + salt) ternary mixtures by refractometry method. J. Chem. Engng Data 51, 1316-1320. 
Molin, D, Mauri, R. \& Tricoli, V. 2007 Experimental evidence of the motion of a single out-of-equilibrium drop. Langmuir 23 (14), 7459-7461.

MoRITA, K. 2013 Effect of distance from equilibrium on spontaneous deformation of droplet driven by Korteweg force. B. Eng. Report, Osaka University.

Nagatsu, Y., BAE, S. K., Kato, Y. \& TADA, Y. 2008b Miscible viscous fingering with a chemical reaction involving precipitation. Phys. Rev. E 77, 067302.

Nagatsu, Y., Hayashi, A., BAn, M., Kato, Y. \& TADA, Y. $2008 a$ Spiral pattern in a radial displacement involving a reaction-producing gel. Phys. Rev. E 78, 026307.

Nagatsu, Y., Matsuda, K., Kato, Y. \& TAdA, Y. 2007 Experimental study on miscible viscous fingering involving viscosity changes induced by variations in chemical species concentrations due to chemical reactions. J. Fluid Mech. 571, 475-493.

Nittmann, J., Daccord, G. \& Stanley, H. E. 1985 Fractal growth viscous fingers: quantitative characterization of a fluid instability phenomenon. Nature 314, 141-144.

ORR, F. M. J. \& TABER, J. J. 1984 Use of carbon dioxide in enhanced oil recovery. Science 224, $563-569$.

Paterson, L. 1985 Fingering with miscible fluids in a Hele-Shaw cell. Phys. Fluids 28, 26-30.

Poesio, P., Beretta, G. P. \& Thorsen, T. 2009 Dissolution of a liquid microdroplet in a nonideal liquid-liquid mixture far from thermodynamic equilibrium. Phys. Rev. Lett. 103, 064501.

Pojman, J. A., Whitmore, C., Liveri, M. L. T., Lombardo, R., Marszalek, J., Parker, R. \& ZolTOWSKI, B. 2006 Evidence for the existence of an effective interfacial tension between miscible fluids: isobutyric acid-water and 1-butanol-water in a spinning-drop tensiometer. Langmuir 22, 2569-2577.

Porter, D. A., EAsterling, K. E. \& Sherif, M. Y. 2009 Phase Transformations in Metals and Alloys, 3rd edn. pp. 302-308. CRC Press.

Pramanik, S. \& Mishra, M. 2013 Linear stability analysis of Korteweg stresses effect on miscible viscous fingering in porous media. Phy. Fluids 25, 074104.

Pramanik, S. \& Mishra, M. 2015 Nonlinear simulations of miscible viscous fingering with gradient stresses in porous media. Chem. Engng Sci. 122, 523-532.

SARgantanis, I. G. \& KARIM, M. N. 1997 Prediction of aqueous two-phase equilibrium using the Flory-Huggins model. Ind. Engng Chem. Res. 36, 204-211.

Schneider, T., Kreutz, J. \& ChiU, D. T. 2013 The potential impact of droplet microfluidics in biology. Anal. Chem. 85, 3476-3482.

Siggia, E. D. 1979 Late stages of spinodal decomposition in binary mixtures. Phys. Rev. A 20, 595-605.

Snyder, S. M., Cole, K. D. \& SzIAG, D. C. 1992 Phase compositions, viscosities, and densities for aqueous two-phase systems composed of polyethylene glycol and various salts at $25^{\circ} \mathrm{C}$. J. Chem. Engng Data 37, 268-274.

Thiam, A. R., Farese, R. V. \& Walther, T. C. 2013 The biophysics and cell biology of lipid droplets. Nat. Rev. Mol. Cell Biol. 14, 775-786.

Truzzolillo, D., Mora, S., Dupas, C. \& Cipelletti, L. 2014 Off-equilibrium surface tension in colloidal suspensions. Phys. Rev. Lett. 112, 128303.

Truzzolillo, D., Mora, S., Dupas, C. \& Cipelletti, L. 2016 Nonequilibrium interfacial tension in simple and complex fluids. Phys. Rev. X 6, 041057.

Vladimirova, N., Malagoli, A. \& Mauri, R. 1999 Diffusiophoresis of two-dimensional liquid droplets in a phase-separating system. Phys. Rev. E 60, 2037-2044. 